\title{
Numerical and Theoretical Analysis of an Alternate Multiwavelength Mode-Locked Fiber Laser
}

\author{
Jérôme Vasseur, Marc Hanna, John M. Dudley, Member, IEEE, and John R. Barry, Senior Member, IEEE
}

\begin{abstract}
The generation of alternate multiwavelength pulse trains in an actively mode-locked erbium-doped fiber laser containing an unbalanced Mach-Zehnder interferometer is analyzed using numerical simulations and a theoretical Gaussian pulse analysis. The results obtained allow the interpretation of recent experimental results reporting multiwavelength pulse laser operation and provide a clear physical description of the operation of the multiwavelength laser. Furthermore, the analysis and modeling also provide guidelines for experimental design.
\end{abstract}

Index Terms-Mode-locked lasers, optical communications.

\section{INTRODUCTION}

$\mathbf{T}$ HE NEED to increase long-haul network performance has led to intense interest in multiwavelength pulsed laser sources around $1550 \mathrm{~nm}$. Recent research has reported successive pulse train generation at different wavelengths [1]-[3] with these sources expected to find applications such as optical code-division multiple-access networks [4] and photonic analog-to-digital conversion [5].

Recently, we proposed a technique to generate successive pulses at different wavelengths using an actively mode-locked erbium-doped fiber laser where an intracavity unbalanced Mach-Zehnder interferometer (UMZI) was used both as an intensity modulator (IM) and a tunable filter, resulting in a new mode-locking mechanism [6]. While similar experimental ideas have recently been reported [7], no detailed theoretical basis for its operation has to date been developed. In this letter, we present both a numerical study of such a multiwavelength source and a complementary steady state Gaussian pulse analysis. As well as providing important physical insight into the laser operation, our results permit a qualitative comparison with the experiments in [6] and provide some useful guidelines for experimental design.

\section{NUMERICAL SimULATIONS}

Fig. 1 shows the laser cavity which contains an erbium-doped fiber amplifier (EDFA), a polarization controller, a 50/50 output coupler, and a UMZI, with a fixed optical path difference of $40 \mu \mathrm{m}$ between its two arms. With no modulating signal, the UMZI acts as a spectral filter of free-spectral range $60 \mathrm{~nm}$. When a radio-frequency signal is applied, its frequency transfer function shifts accordingly and acts as a tunable filter. For a sinu-

Manuscript received May 9, 2005; revised July 19, 2005.

J. Vasseur and M. Hanna are with GTL-CNRS Telecom, UMR CNRS 6174 , Metz 57070, France (e-mail: jvasseur@georgiatech-metz.fr).

J. M. Dudley is with the Laboratoire FEMTO-ST, UMR CNRS 6174, Université de Franche-Comté, Besançon 25030 Cedex, France.

J. R. Barry is with the Department of Electrical and Computer Engineering, Georgia Institute of Technology, Atlanta, GA 30332 USA

Digital Object Identifier 10.1109/LPT.2005.857608

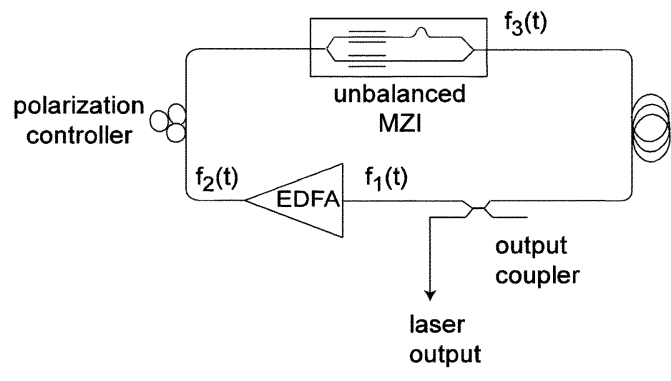

Fig. 1. Schematic diagram of the alternate multiwavelength laser.

soidal modulation signal, for a fixed wavelength, it also plays the role of IM, generating pulses via a mode-locking operation. Each wavelength sees a different temporal transfer function, leading to the alternate production of pulses at different wavelengths.

Numerical simulations of the laser are carried out by injecting a weak noise field and allowing it to numerically evolve to a steady state subject to the action of the various components shown in Fig. 1. The fiber propagation is modeled using the split-step Fourier method [8]. Amplifier gain saturation is modeled as

$$
g=\frac{g_{0}}{1+P_{m} / P_{\text {sat }}}
$$

where the small signal gain $g_{0}$ is $30 \mathrm{~dB}, P_{m}$ is the mean power of the input signal and the saturation power at the EDFA input $P_{\text {sat }}$ is $-10 \mathrm{dBm}$. The amplified spontaneous noise of the EDFA is considered to be white and Gaussian. The EDFA gain curve is modeled by a Gaussian function of bandwidth $30 \mathrm{~nm}$. Being in a push-pull configuration, the UMZI output $Y(t)$ is related to its input $X(t)$ by

$$
Y(t)=\frac{1}{2}\left[X\left(t-\frac{\tau(t)}{2}\right)+X\left(t+\frac{\tau(t)}{2}\right)\right]
$$

where $\tau(t)$ is the delay introduced by the interferometer. It is assumed to be of the form $\tau(t)=\tau_{0}+\Delta \tau \sin (\Omega t)$, where $\Omega$ is the modulation frequency of the UMZI. We choose $\omega_{0}$ to be a maximum of transmission for the filter at $t=0\left(\omega_{0}=4 \mathrm{k} \pi / \tau_{0}\right.$, with $k$ an integer). The UMZI is modeled using a discretized version of (2).

Fig. 2(a) and (b) shows the simulated temporal trace and spectrum of the laser output for parameters: $\tau_{0}=1.33 \sim 10^{-13}$ $\mathrm{s}, \omega_{0}=1.216 \sim 10^{15} \mathrm{rad} / \mathrm{s}, \Omega=7.18 \sim 10^{10} \mathrm{rad} / \mathrm{s}$, $\Delta \tau=8.52 \sim 10^{-16} \mathrm{~s}$, and $\Delta \omega=23.55 \sim 10^{12} \mathrm{rad} / \mathrm{s}$. Here, the dispersion in the cavity is $0 \mathrm{ps} / \mathrm{nm} / \mathrm{km}$. The round-trip cavity length is $20 \mathrm{~m}$, and the steady state results shown were obtained after 200 loops. For this repetition rate of $11.33 \mathrm{GHz}$, two successive trains at two different wavelengths $\left(\lambda_{1}=1540 \mathrm{~nm}\right.$ and $\lambda_{2}=1560 \mathrm{~nm}$ ) are observed. The pulsewidths are 7 and 

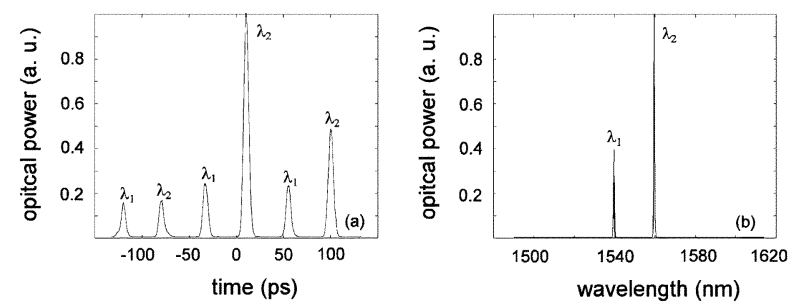

Fig. 2. (a) Time trace and (b) spectrum of the laser output obtained by simulation.
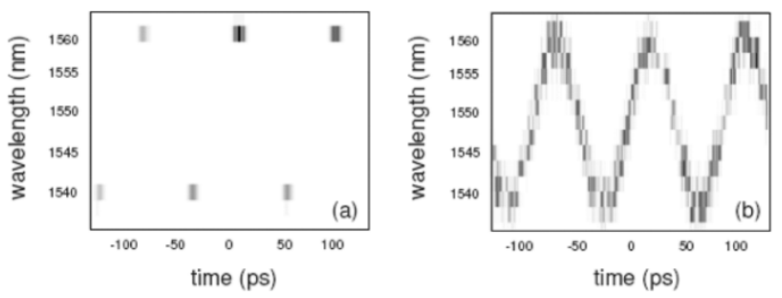

Fig. 3. Spectrograms obtained by simulation with (a) a Gaussian and (b) an infinitely flat gain medium.

$6.5 \mathrm{ps}$, yielding time-bandwidth products (TBPs) of 0.72 and 0.61 , respectively. The alternate multiwavelength qualitative behavior observed in [6] is reproduced. The spectral flatness could be improved by adding a gain-equalizing filter in the cavity.

The multiwavelength pulsed laser operation is conveniently examined in the time-frequency domain through the pulse spectrogram [9]. Fig. 3(a) shows the spectrogram for the results in Fig. 2. In comparison, Fig. 3(b) shows the spectrogram obtained when the gain is assumed to be infinitely flat rather than Gaussian, and we see continuous emission with varying frequency. In this case, the UMZI is the only frequency-selective element in the cavity, and thus, imposes the emitted wavelength through the evolution of the maximum $\omega_{\max }$ of its transfer function [6]

$$
\omega_{\max }(t) \approx \omega_{0}\left(1-\frac{\Delta \tau}{\tau_{0}} \sin (\Omega t)\right) .
$$

This observation indicates that the filtering action of the UMZI dominates in ensuring multiwavelength operation, but the detailed time-wavelength characteristics observed depend on the interaction of the UMZI with the particular gain profile of the EDFA. The general multiwavelength behavior observed would be expected with any continuous gain profile.

Numerical simulations were also performed with a conventional IM added in the cavity to control the pulse timing [6]. Synchronously driven at an integer multiple $N$ of the UMZI modulation frequency, it locks the emission of pulses on a temporal grid, while the central wavelength is determined by the UMZI. Fig. 4 shows simulation results after 200 loops, when the modulation frequency of the IM $\left(f_{\mathrm{im}}=\Omega_{\mathrm{im}} / 2 \pi=34 \mathrm{GHz}\right)$ is three times the modulation frequency of the UMZI ( $\left.f_{\mathrm{UMZI}}=\Omega / 2 \pi=11.33 \mathrm{GHz}\right)$. The other parameters are set to: $\omega_{0}=1.216 \sim 10^{15} \mathrm{rad} / \mathrm{s}, \tau_{0}=1.33 \sim 10^{-13} \mathrm{~s}$, $\Delta \tau=1.29 \sim 10^{-15} \mathrm{~s}, \Delta \tau_{\mathrm{im}}=1.29 \sim 10^{-15} \mathrm{~s}$, and $\Delta \omega=23.5 \sim 10^{12} \mathrm{rad} / \mathrm{s}$.
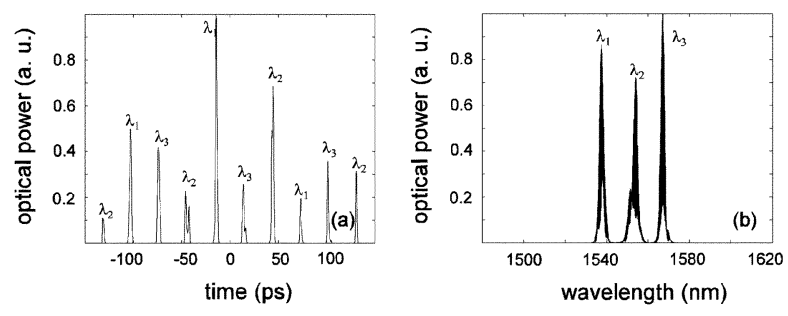

Fig. 4. Simulation results in the presence of an additional IM in the (a) time domain and (b) spectral domain.

Three series of pulses at three different wavelengths $\left(\lambda_{1}=\right.$ $1538 \mathrm{~nm}, \lambda_{2}=1554 \mathrm{~nm}$, and $\lambda_{3}=1567 \mathrm{~nm}$ ) are obtained. Two successive pulses are separated by $1 / f_{\text {im }}$ and two pulses at the same wavelength by $1 / f_{\mathrm{UMZI}}$, as expected. The pulse widths are recorded to be $1.9,2.5$, and $1.7 \mathrm{ps,} \mathrm{yielding} \mathrm{a} \mathrm{TBP} \mathrm{of} 0.57$, 0.78 , and 0.51 .

The numerical simulations reproduce well the qualitative behavior of the multiwavelength source that has been reported in experiments. To gain even further insight into the laser operation, a theoretical model using a Gaussian pulse analysis [10] can be used to examine the pulse characteristics at a particular operating point. This model will be compared with the experimental results obtained in [6].

\section{GAUSSIAN ANALYSIS}

The steady state solution is assumed to be described by a linearly chirped complex Gaussian envelope

$$
f_{1}(t)=A \exp \left(i \omega_{0} t-\Gamma t^{2}\right)
$$

where $\Gamma=\alpha_{1}-i \beta_{1}$ is the complex Gaussian pulse parameter and $\omega_{0}$ the angular reference frequency. The pulse propagates through the EDFA, modeled as a Gaussian spectral transfer function

$$
g(\omega)=\exp \left(-\frac{\left(\omega-\omega_{0}\right)^{2}}{\Delta \omega^{2}}\right)
$$

where $\Delta \omega$ is the gain bandwidth. At the ouput of the gain medium, the resulting pulse is

$$
f_{2}(t)=C \exp \left(i \omega_{0} t-\Gamma^{\prime} t^{2}\right)
$$

with

$$
\begin{aligned}
\Gamma^{\prime} & =\frac{\left(\Delta \omega^{4} \alpha_{1}+4 \Delta \omega^{2}\left(\alpha_{1}^{2}+\beta_{1}^{2}\right)\right)-i \beta_{1} \Delta \omega^{4}}{\left(\Delta \omega^{2}+4 \alpha_{1}\right)^{2}+\left(4 \beta_{1}\right)^{2}} \\
& =\alpha_{1}^{\prime}-i \beta_{1}^{\prime}
\end{aligned}
$$

The pulse then goes through the UMZI. Assuming a Gaussian input pulse, and since the delay introduced by the modulator is small compared to the pulse characteristic times $\alpha_{1}^{-1 / 2}$ and $\beta_{1}^{-1 / 2}$, the temporal transfer function of the UMZI can be approximated by a Gaussian function in the neighborhood of $t=$ 0 , and the pulse at the UMZI output can be written as

$$
f_{3}(t)=f_{2}(t) T_{\alpha_{1}^{\prime}, \beta_{1}^{\prime}}(t)
$$



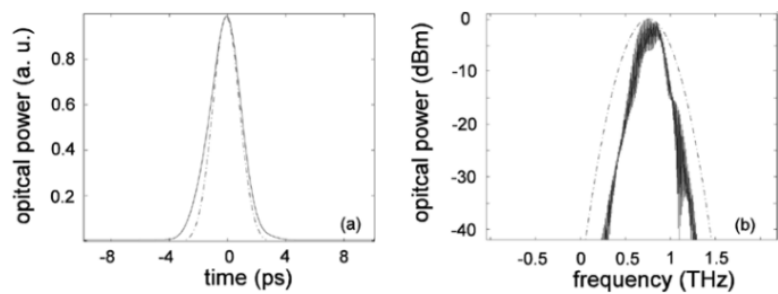

Fig. 5. (a) Temporal and (b) spectral pulse shape obtained by simulation (solid line) and theory (dotted line).

Here, $T_{\alpha^{\prime} 1, \beta^{\prime} 1}(t)$ is a transfer function valid around $t=0$ when the filter transmission is at midpoint of the swept wavelength range and where the chirp is maximum

$$
T_{\alpha_{1}, \beta_{1}}(t) \approx \exp \left[-\frac{1}{2}\left(\frac{\omega_{0} \Delta \tau \Omega}{2}+\tau_{0}\left(\beta_{1}+i \alpha_{1}\right)\right)^{2} t^{2}\right] .
$$

Imposing the steady-state condition $f_{3}(t)=f_{1}(t)$ gives

$$
\left\{\begin{array}{l}
\alpha_{1}=\frac{\omega_{0} \Delta \tau \Omega}{\tau_{0}} \sqrt{\frac{2}{8+\tau_{0}^{2} \Delta \omega^{2}}} \\
\beta_{1}=-\frac{\omega_{0} \Delta \tau \Omega}{2 \tau_{0}}
\end{array}\right.
$$

The nonzero value of $\beta_{1}$ indicates that the pulses are chirped. The full-widths at half-maximum of the pulse in the time and spectral domain can be retrieved from $\alpha_{1}$ and $\beta_{1}$ in a straightforward manner. As expected, the pulsewidth decreases when the modulation frequency increases, which is in agreement with the experimental results in [6]. We can note that if the gain medium is infinitely flat, the value for $\beta_{1}$ does not change, whereas $\alpha_{1}$ becomes zero, indicating a continuous laser emission with variable frequency, as observed in simulations (Fig. 3). By differentiating (3), we verify that both approaches are consistent

$$
\left|\frac{d \omega_{\max }}{d t}\right|_{t=0}=2\left|\beta_{1}\right|
$$

Fig. 5(a) and (b) displays a comparison of simulations and analytical results for the laser output around $t=0$. The parameters are chosen to be: $\tau_{0}=1.33 \sim 10^{-13} \mathrm{~s}, \omega_{0}=1.216 \sim$ $10^{15} \mathrm{rad} / \mathrm{s}, \Omega=7.12 \sim 10^{10} \mathrm{rad} / \mathrm{s}, \Delta \tau=1.29 \sim 10^{-15} \mathrm{~s}$, and $\Delta \omega=21.35 \sim 10^{12} \mathrm{rad} / \mathrm{s}$. The simulated pulsewidth is recorded to be $2.3 \mathrm{ps}$ in the temporal domain and $310 \mathrm{GHz}$ in the frequency domain, yielding a TBP of 0.71 . Equation (11) predicts pulsewidths of $2.1 \mathrm{ps}$ and $350 \mathrm{GHz}$, i.e., a TBP of 0.76 . As expected, the produced pulses are chirped and theory and simulations are in a good agreement.

The previous theoretical analysis can be modified to incorporate the effect of the IM. In this case, the resulting pulse parameters are found to be

$$
\left\{\begin{array}{l}
\alpha_{1}=\frac{\omega_{0}}{\sqrt{8+\tau_{0}^{2} \Delta \omega^{2}}}\left[\frac{2 \Delta \tau^{2} \Omega^{2}}{\tau_{0}^{2}}+\frac{\Delta \omega^{2} \Delta \tau_{\mathrm{im}}^{2} \Omega_{\mathrm{im}}^{2}}{4}\right]^{\frac{1}{2}} \\
\beta_{1}=-\frac{\omega_{0} \Delta \tau \Omega}{2 \tau_{0}}
\end{array}\right.
$$

where $\Omega_{\mathrm{im}}=N \Omega$ is the modulation frequency of the IM and $\Delta \tau_{\text {im }}$ its modulation depth. It is interesting to notice that the addition of the IM does not change the value of $\beta_{1}$, viz. of the chirp parameter, but only the value of $\alpha_{1}$, and thus, of the pulsewidth. This indicates that the chirp is solely due to the frequency sweeping of the UMZI.

This model can also be compared with the experimental results obtained in [6]: Three pulse trains at three different wavelengths $(1543.2,1549.6$, and $1562.5 \mathrm{~nm})$, each at a repetition rate of $3.8 \mathrm{GHz}$, were generated. The time traces were measured to be 24,25 , and 38 ps on the $30-\mathrm{GHz}$ oscilloscope. Assuming that both the optical pulses and the detector impulse response are Gaussian, this leads to pulsewidths of 17, 18, and $34 \mathrm{ps}$ and TBPs of $0.57,0.77$, and 1.65 , respectively. Theory predicts 3.1-ps pulses with a TBP of 0.76 . In fact, the theoretical and numerical results presented here allow us to understand the qualitative behavior observed in these experiments, and to understand the physical origin of the chirp that was reported on the experimental pulses. However, some quantitative differences remain, with the measured pulse durations being significantly longer than those predicted from our modeling. The intensities of the lasing wavelengths also differ. We attribute this difference to approximations in the gain model used for the EDFA and uncertainties in the linear dispersive properties of the various elements used in the laser shown in Fig. 1. These specific points will require further study to obtain a quantitatively accurate model of the laser.

\section{CONCLUSION}

We have numerically and theoretically analyzed the generation of time- and wavelength-interleaved mode-locked pulse trains. This laser uses a UMZI in an erbium-doped fiber ring cavity as a mode-locker and a tunable filter. Additional timing control of the emitted pulses using an additional IM in the cavity was also studied theoretically. Our analysis provides good physical understanding of the laser operation, and reproduces qualitatively results obtained in previous experiments.

\section{REFERENCES}

[1] Q. Mao and J. W. Y. Lit, "Multiwavelength erbium-doped fiber lasers with active overlapping linear cavities," J. Lightw. Technol., vol. 21, no. 1, pp. 160-169, Jan. 2003.

[2] J. Sun and W. Liu, "Multiwavelength generation by utilizing second-order nonlinearity of $\mathrm{LiNbO}_{3}$ waveguides in fiber lasers," Opt. Commun., vol. 224, pp. 125-130, 2003.

[3] J. Yao, Y. Wang, S. C. Tjin, Y. Zhou, Y. L. Lam, J. Liu, and C. Lu, "Active modelocking of tunable multi-wavelength fiber ring laser," Opt. Commun., vol. 191, pp. 341-345, 2001.

[4] J. A. Salehi, "Code division multiple-access techniques in optical fiber networks-Part I: Fundamental principles," IEEE Trans. Commun., vol. 37, no. 8, pp. 824-833, Aug. 1989.

[5] A. Yariv and R. G. M. P. Koumans, "Time interleaved optical sampling for ultrahigh speed A/D conversion," Electron. Lett., vol. 34, pp. 2012-2013, 1998.

[6] J. Vasseur, M. Hanna, J. Dudley, and J. P. Goedgebuer, "Alternate multiwavelength modelocked fiber laser," IEEE Photon. Technol. Lett., vol. 16, no. 8, pp. 1816-1818, Aug. 2004.

[7] R. Huber, K. Taira, and J. G. Fujimoto, "Fourier domain mode locking: Overcoming limitations of the frequency swept light sources and pulsed lasers," in CLEO Eur.-EQEC Conf., Munich, 2005, Postdeadline Paper CP3-6-THU.

[8] G. P. Agrawal, Nonlinear Fiber Optics. New York: Academic, 2001.

[9] R. Trebino, Frequency-Resolved Optical Gating: The Measurement of Ultrashort Laser Pulses. Norwell, MA: Kluwer, 2000.

[10] A. Yariv, Optical Electronics in Modern Communications. London, U.K.: Oxford Univ. Press, 1997. 